\title{
Bronquiectasias: ¿No es más una enfermedad huérfana?
}

\author{
PATRICIA FERNÁNDEZ V.*** y PATRICIO JIMÉNEZ P.**
}

\section{Bronchiectasis: Is it no longer considered an orphan disease?}

Bronchiectasis, so far considered an orphan disease, currently is diagnosed with a higher frequency due to several reasons such as renewed awareness of the disease, better diagnosis including imagenology, the development of patients registries, as well as a higher number of clinical research studies. The pathological basis of bronchiectasis is widely variable. Also the clinical expression is variable, from absence of symptoms in some patients up to chronic sputum production in others. Furthermore, a group of patients often develop recurrent exacerbations. Despite the etiologies of bronchiectasis are diverse, the main etiology is previous pulmonary infection. On the other hand, bronchiectasis could also be the expression of diverse systemic diseases. Even around one quarter of patients the etiology would not be established. The development of large registries of patients has allowed the building of classifications systems with accurate prognostic criteria. Chronic infection is the most relevant issue in bronchiectasis. Infection with P. aeruginosa has been associated with poor prognosis and their eradication must be attempted always. Effective secretions drainage techniques, oral and nebulized antibiotics, as well as mucolytic therapy are the mainstay of treatment in bronchiectasis.

Key words: Bronchiectasis; antibacterial agents; Pseudomonas aeruginosa; respiratory tract infections.

\section{Resumen}

Las bronquiectasias, consideradas hasta un tiempo atrás una enfermedad huérfana, se diagnostican actualmente con mayor frecuencia debido a un renovado interés en esta patología, a una mejoría de técnicas de diagnóstico, existencia de mejores registros, acceso a mejores imágenes y aumento de los estudios clínicos. El sustrato anátomo-patológico es notablemente variable, al igual que la expresión clínica, que va desde la ausencia de síntomas hasta la presencia de broncorrea crónica. A su vez, un grupo de pacientes tiende a presentar exacerbaciones frecuentes. Las etiologías de las bronquiectasias son múltiples, siendo la más frecuente la existencia de infecciones pulmonares previas. También pueden formar parte de enfermedades crónicas sistémicas. Sin embargo, en 25\% de los casos no es posible identificar la etiología. El desarrollo de registros de pacientes ha permitido construir modelos de clasificación de gravedad, lo que hace posible establecer criterios pronósticos. La infección crónica es un hecho frecuente en bronquiectasias y la presencia de P. aeruginosa confiere mal pronóstico a la enfermedad. La erradicación de Pseudomonas debe ser intentada siempre en estos pacientes. El uso de técnicas de drenaje, los antibióticos orales y nebulizados y las terapias mucolíticas constituyen los pilares centrales en el manejo de la enfermedad.

Palabras clave: Bronquiectasias, agentes antibacterianos; Pseudomonas aeruginosa; infecciones del tracto respiratorio.

\section{Introducción}

Muchos autores en el siglo XXI aún siguen describiendo que las bronquiectasias son una enfermedad rara. Nosotros podemos argumentar que las bronquiectasias conforman una enfermedad común, pero poco estudiada y ésta es quizás la razón por la cual el término de enfermedad huérfana todavía puede ser un término apropiado.

A esta enfermedad y a los pacientes se les debe

* Servicio de Medicina, Instituto Nacional del Tórax.

** Clínica Santa María. Santiago de Chile. 
posicionar en el lugar que les corresponde por la importancia de esta patología. Es sorprendente cómo en los últimos años se está trabajando en registros nacionales y en estudios clínicos en esta materia.

Según la definición de la Unión Europea, se denomina enfermedades huérfanas a aquellas en las cuales la prevalencia es menor de 5/10.000 habitantes; por lo tanto, las bronquiectasias no podrían definirse como una enfermedad huérfana ya que su prevalencia en USA fluctúa entre 52 a 370/100.000 habitantes y en Alemania alcanza a 67/100.000 habitantes ${ }^{1}$. Este reporte señala que tanto la prevalencia como la incidencia van en permanente aumento, lo que junto al incremento del diagnóstico, puede deberse a varios factores: mejoría en técnicas de diagnóstico (por ejemplo, mayor disponibilidad de la tomografía computarizada), mayor estudio de la enfermedad y nuevas técnicas inmunológicas y microbiológicas.

Está claro que con la creciente evidencia mucho queda por descubrir bajo el agua ya que lo que vemos son sólo los pacientes registrados, no conociendo los pacientes leves o moderados que se atienden en un sistema de salud primario, tampoco conocemos la cantidad de pacientes con signos y síntomas, pero sin un diagnóstico radiológico y menos aún aquellos asintomáticos a quienes nunca se le ha realizado una radiografía.

\section{Heterogeneidad de las bronquiectasias}

Al tratarse de una enfermedad secundaria a un desorden morfológico, el diagnóstico generalmente comienza por una imagen radiológica, con una diversidad que comprende las bronquiectasias cilíndricas, varicosas, quísticas, localizadas y difusas (Figura 1). Por otra parte, desde el punto de vista clínico los pacientes con bronquiectasias pueden presentarse con signos y síntomas diversos, que incluye a los tosedores productivos crónicos, los exacerbadores frecuentes, y los que debutan con hemoptisis, incluyendo además a pacientes totalmente asintomáticos.

Por otra parte, si evaluamos la función pulmonar, podemos clasificarlos en obstructivos leves, moderados y severos. Si los clasificamos con respecto a la colonización, entonces ¿por qué algunos se colonizan con Pseudomonas aerugino$s a$, otros con estafilococos aureus, algunos con $H$. influenzae y otros nunca se colonizan?
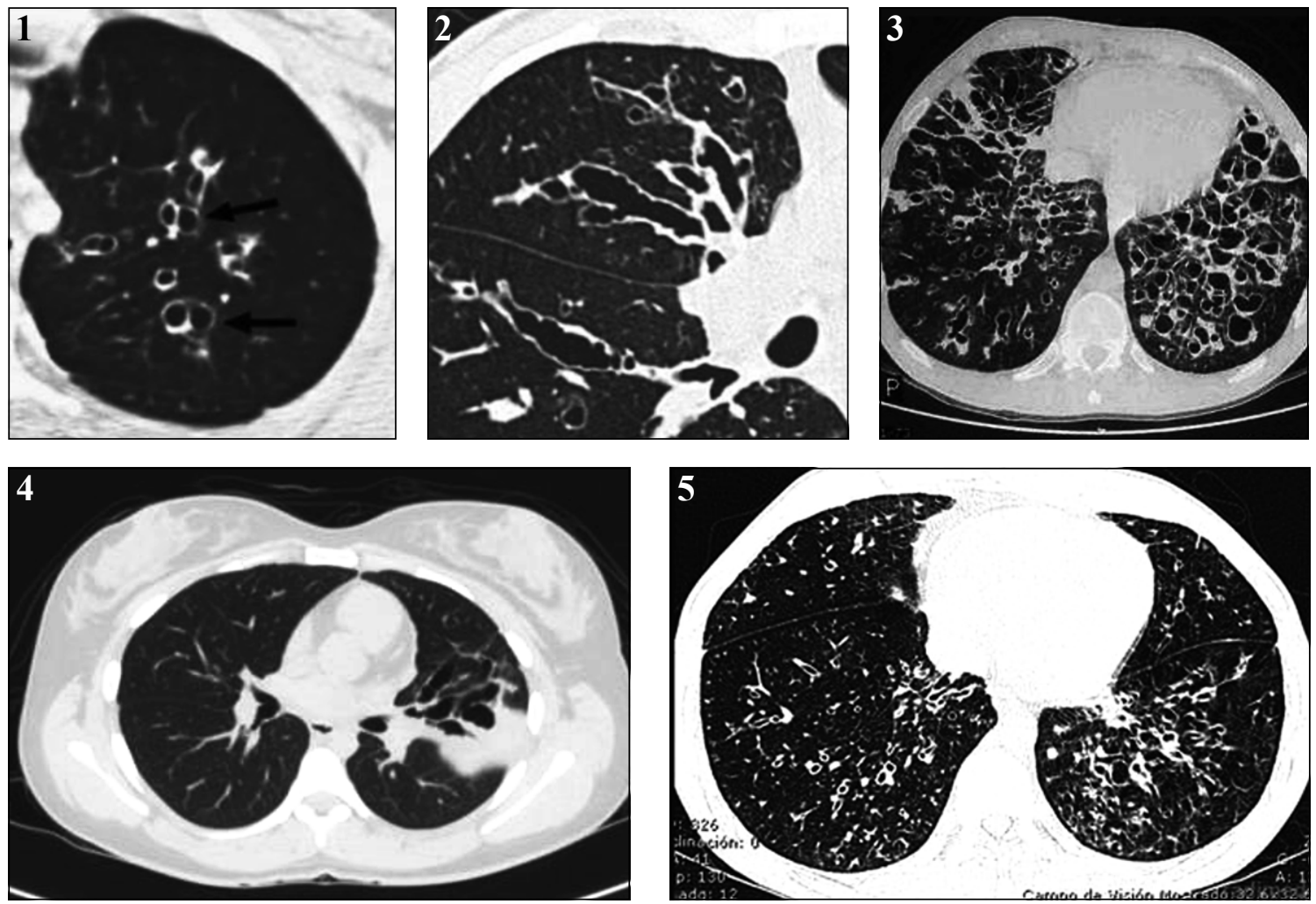

Figura 1. Imágenes de bronquiectasias en la tomografía axial computarizada (TAC). 1: bronquiectasias cilíndricas; 2: bronquiectasias varicosas; 3: bronquiectasias quísticas; 4: bronquiectasias localizadas; 5: bronquiectasias difusas. 

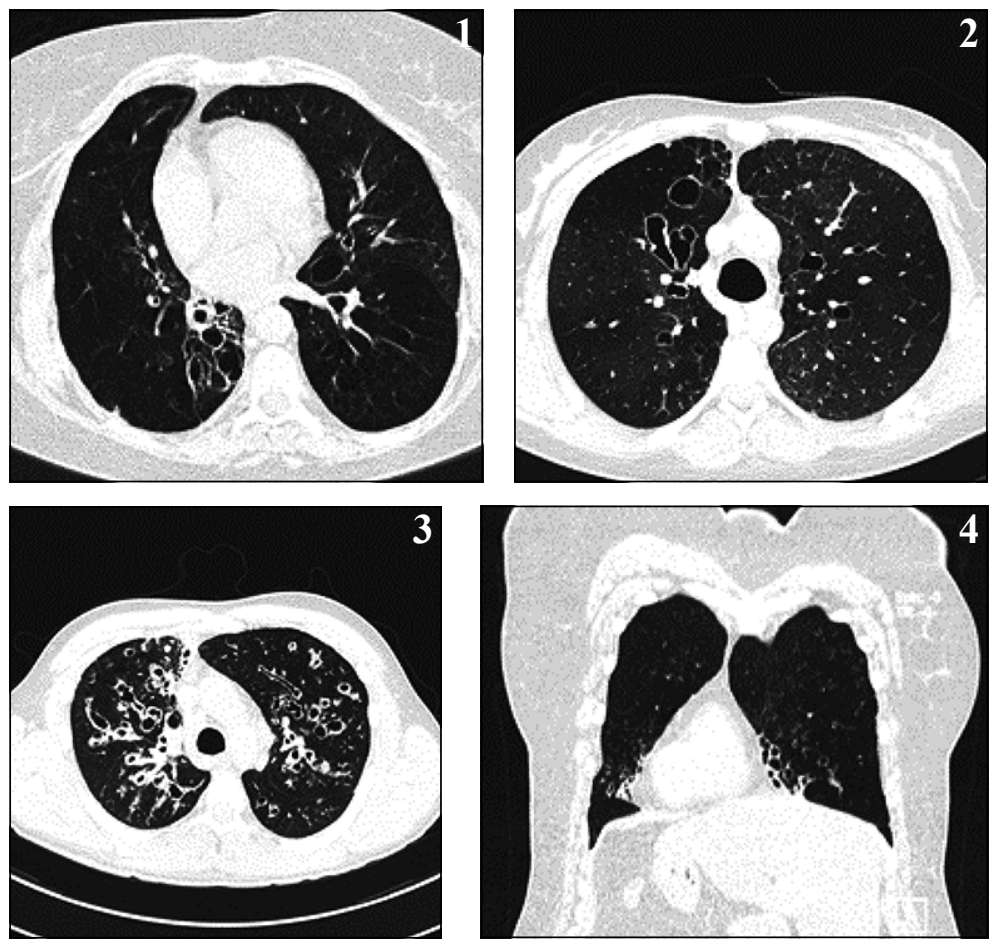

Figura 2. Imágenes de bronquiectasias de diversas etiologías en la TAC. 1: Post TBC; 2: Post adenovirus; 3: Fibrosis quística; 4: Síndrome de Kartagener.

\section{Etiología de las bronquiectasias (BQ)}

Hoy día sabemos que existen muchas enfermedades que pueden causar BQ (Figura 2), siendo la más frecuente la etiología post-infecciosa; sin embargo, pese a todos los avances en el diagnóstico hasta en el $25 \%$ de las BQ no se puede establecer su etiología ${ }^{2}$ (Tabla 1).

Las BQ post infecciosas son generalmente secundarias a procesos como tuberculosis, adenovirus en la infancia, coqueluche y sarampión.

Las BQ también están presentes en anomalías mucociliares como la fibrosis quística (FQ), consistente en una mutación en el cromosoma 7, que aparece precozmente en la vida, generalmente acompañada de sinusitis, insuficiencia pancreática y azoospermia, cuyo diagnóstico, además de la sospecha clínica, se debe confirmar con test del sudor o con estudio genético. Otra alteración es la disquinesia ciliar o síndrome de cilio inmóvil, desorden genético que provoca una alteración de la estructura y función del cilio, acompañado de sinusitis e infertilidad, cuyo diagnóstico se hace por biopsia y microscopía electrónica; también se describe el test de sacarina como técnica de diagnóstico, con menos sensibilidad y especificidad. Si esta patología se acompaña de situs inverso se le denomina síndrome de Kartagener.

El déficit de alfa-1 antitripsina, enfermedades
Tabla 1. Etiología de las bronquiectasias*

\begin{tabular}{|ll|}
\hline $\begin{array}{l}\text { Obstrucción de la } \\
\text { vía aérea }\end{array}$ & $\begin{array}{l}\text { Cuerpo extraño bronquial } \\
\text { Tumor carcinoide, condroma } \\
\text { Tapones mucosos }\end{array}$ \\
\hline Post infecciosa & $\begin{array}{l}\text { Adenovirus } \\
\text { Sarampión } \\
\text { B. pertussis } \\
\text { Tuberculosis }\end{array}$ \\
$\begin{array}{l}\text { Anormalidades } \\
\text { mucociliares }\end{array}$ & $\begin{array}{l}\text { Fibrosis quística } \\
\text { Disquinesia ciliar }\end{array}$ \\
$\begin{array}{l}\text { Anormalidades } \\
\text { inmunológicas }\end{array}$ & $\begin{array}{l}\text { Hipogamaglobulinemia } \\
\text { Anormalidad función neutrófilos }\end{array}$ \\
$\begin{array}{l}\text { Enfermedades } \\
\text { sistémicas }\end{array}$ & $\begin{array}{l}\text { Artritis reumatoide } \\
\text { Síndrome de Sjögren } \\
\text { Espóndiloartritis anquilosante } \\
\text { Déficit de } \alpha-1 \text { antitripsina }\end{array}$ \\
\hline EPOC & Avanzada (VEF ${ }_{1}$ 50-30\%) \\
\hline
\end{tabular}

*Adaptado de McShane et al. ${ }^{2}$

inflamatorias intestinales como la colitis ulcerosa (inflamación no granulomatosa de vía aérea) y la enfermedad de Crohn (inflamación granulomatosa de vía aérea), así como el síndrome de Sjögren y la Artritis Reumatoide (AR) también son causas de BQ. La aspergilosis broncopulmonar alérgica (ABPA) produce BQ centrales. Otra etiología a considerar son las inmunodeficiencias. 
Goeminne y cols., analizaron una serie de casos extraídos de publicaciones inglesas, chinas y norteamericanas, y concluyeron que la etiología más frecuente es la idiopática (30 a $66 \%$ ), seguida por la post-infecciosa (19 a 43\%), inmunodeficiencias (1 a 9\%), EPOC (1 a 36\%), enfermedades del tejido conectivo como Sjögren y AR (1 a $11 \%)$, enfermedad inflamatoria intestinal (1 a 3\%), reflujo gastroesofágico (RGE) (1 a $4 \%)$ y $\operatorname{ABPA}(1 \text { a } 8 \%)^{3}$.

\section{Clasificación de gravedad de las bronquiectasias}

Convencionalmente el volumen espiratorio forzado en el primer segundo $\left(\mathrm{VEF}_{1}\right)$ es usado como índice de la severidad de la enfermedad. Sin embargo, hoy sabemos que existen otros factores que están directamente relacionados con la gravedad de la enfermedad.

Existen dos puntajes de gravedad para clasificar los pacientes con BQ según su riesgo. Chalmers y cols., introdujeron el índice de severidad de BQ (BSI), que consiste en asignar puntajes a la TAC de tórax, $\mathrm{VEF}_{1}$, disnea medida mediante la escala modificada del Medical Research Cou$n c i l$ (mMRC), colonización por P. aeruginosa, exacerbaciones y hospitalizaciones previas ${ }^{4}$.

El puntaje de severidad de FACED, que personalmente consideramos el más simple y fácil de realizar, fue publicado por Martínez García estudiando 819 pacientes con BQ no fibrosis quística (FQ) seguidos durante cinco años. Considera cinco variables a saber: función pulmonar, edad, colonización, extensión y disnea ${ }^{5,6}$. La puntuación de cada variable y el valor del índice en la cohorte de validación se muestran en la Figura 3.

\section{Exámenes para estudio etiológico de bronquiectasias}

Se recomienda solicitar determinación de IgG, IgA e IgM para descartar inmunodeficiencia, eosinófilos en sangre, IgE sérica y tests específicos para descartar ABPA. Especialmente en niños $\mathrm{y}$ en pacientes trasplantados hay que descartar RGE. En todo niño con BQ y también adultos menores de 40 años con historia de mal absorción intestinal o infertilidad se debe realizar el test del sudor para descartar FQ. Ante sospecha de disquinesia ciliar en paciente con infertilidad y/o sinusitis se debe realizar determinación de FeNO nasal que se encuentra muy bajo o bien test de sacarina; si éste resulta positivo se debe realizar estudio de morfología ciliar mediante biopsia en un centro especializado.

\section{Infección crónica de las bronquiectasias}

La mayoría de los estudios describen que los principales gérmenes colonizantes son: $H$. influenzae (14-52\%), P. aeruginosa (15-58\%), S. pneumoniae (7-37\%) y M. catarralis $(8-27 \%)^{7}$.

De acuerdo a un estudio de McShane publicado en 2013, hay que tener presente que, aun teniendo esputo purulento, en el 18 a $40 \%$ de las muestras no se cultivan patógenos ${ }^{2}$.

Por otra parte, Finch y cols., en el estudio EMBARC (registro de BQ europeo) describen que en los pacientes colonizados con $P$. aeruginosa aumenta tres veces la mortalidad y siete veces el riesgo de hospitalización, tienen una exacerbación más por año y un $\mathrm{VEF}_{1} 15 \%$ más bajo en comparación con aquellos pacientes con BQ no colonizados por $P$. aeruginos $a^{8}$.

\begin{tabular}{|ll|c|}
\hline & & Puntos \\
\hline Colonización con & no & 0 \\
P. aeruginosa & sí & 1 \\
\hline \multirow{2}{*}{ Disnea mMRC } & $0-$ II & 0 \\
& III-IV & 1 \\
\hline \multirow{2}{*}{ VEF $_{1}(\%)$} & $\geq 50$ & 0 \\
& $<50$ & 2 \\
\hline \multirow{2}{*}{ Edad } & $<70$ & 0 \\
\hline Lóbulos & $\geq 70$ & 2 \\
comprometidos & $1-2$ & 0 \\
\hline
\end{tabular}

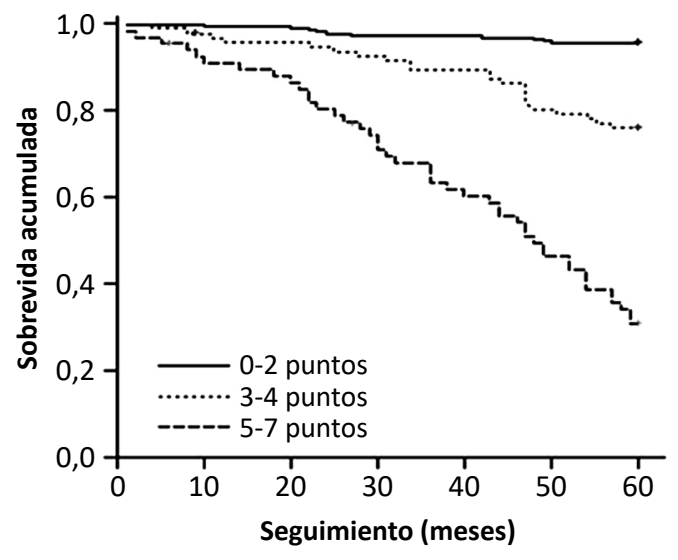

Figura 3. Índice de gravedad de FACED en bronquiectasias no Fibrosis Quística. Adaptado de Martínez-García M, et al5 . 


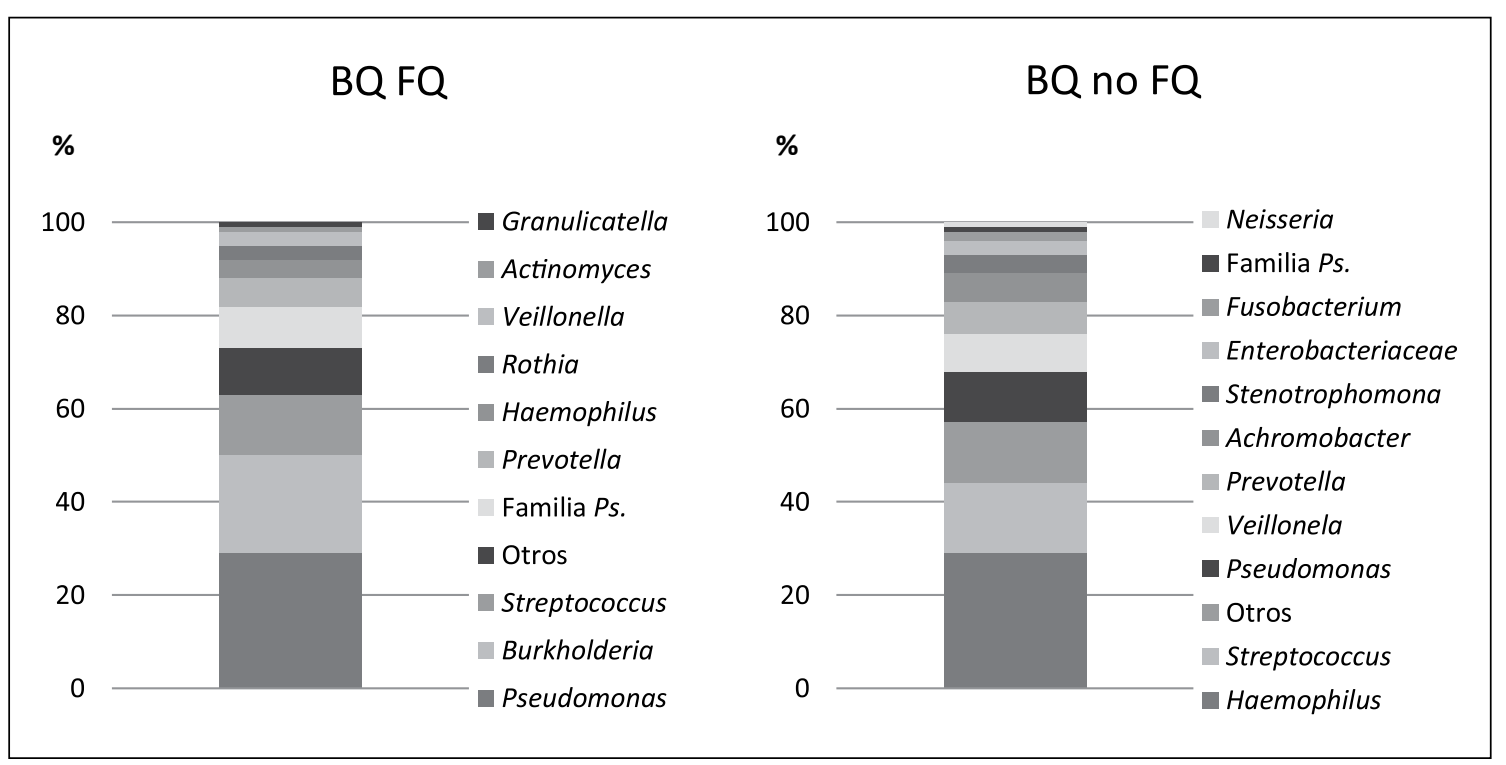

Figura 4. Diferencia microbiana en bronquiectasias en fibrosis quística (BQ FQ) y bronquiectasias no fibrosis quística (BQ no FQ). Pseudomonas y Burkholderia son los gérmenes porcentualmente más frecuentes en BQ FQ, en tanto que Haemophilus y Streptococcus son los predominantes en BQ no FQ.

La prevalencia de colonización con micobacteria no tuberculosa (MNT), en BQ es de 9,3\%; de ellos 53\% son MAC (M. avium complex), 39\% M. abscessus, 3 a $28 \%$ M. kansasii, 1 a $3 \% M$. fortuitum, $1 \% \mathrm{M}$. chelonae y $1 \% \mathrm{M}$. malmoense. Estos casos están asociados a infección por $P$. aeruginosa en el $52 \%$ y generalmente no son exacerbadores frecuentes ${ }^{9}$. No existen datos publicados de infección por MNT en BQ en Chile.

\section{Diferencia microbiana en BQ no FQ y BQ FQ}

Mirando a nivel genético podemos ver que la microbiota presente en bronquiectasias en pacientes no FQ y BQ FQ son muy distintas; mientras en el segundo los patógenos más frecuentes son P. aeruginosa y Burkholderia, en BQ no FQ los patógenos principalmente encontrados son $H$. influenzae y Streptococcus pneumoniae (Figura 4).

\section{Fenotipos clínicos en pacientes con bronquiectasias}

Aliberti y cols., han publicado recientemente un estudio de cinco bases de datos con 1.145 pacientes en el que identifican 4 fenotipos clínicos: Cluster 1: pacientes colonizados con $P$. aeruginosa, Cluster 2: infección crónica con otro microorganismo, Cluster 3: pacientes expectoradores diarios, Cluster 4: bronquiectasias secas ${ }^{10}$.

\section{Manejo de las bronquiectasias}

El objetivo fundamental del tratamiento es reducir las exacerbaciones. Con ello está demostrado que se reduce la mortalidad y disminuyen las hospitalizaciones, mejorando la calidad de vida. Un estudio finlandés señala que las bronquiectasias tienen mejor sobrevida que el asma, pero peor que la EPOC después de la primera hospitalización ${ }^{11}$. Otro estudio reciente australiano muestra que la sobrevida es similar a la población general, pero para ello se necesita un manejo cuidadoso y dedicado de los pacientes ${ }^{12}$. Los pilares del tratamiento residen en tres elementos: mejorar el clearance mucociliar, disminuir o evitar las colonizaciones y tratar la inflamación de la vía aérea.

La revisión sistemática Cochrane de 2015 concluye que los antibióticos pueden reducir la producción de esputo, exacerbaciones y hospitalizaciones; que el tratamiento con corticoides inhalados puede mejorar la función pulmonar, pero su efecto es reducido; que las técnicas de clearance mucociliar pueden reducir el esputo y mejorar la calidad de vida, la asociación LABA ( $\beta$-2 antagonistas de acción prolongada)+corticoides inhalados pueden reducir disnea, sibilancias y tos y finalmente que la $\mathrm{Rh}$-DNasa no muestra beneficio, como sí ocurre en pacientes con fibrosis quística ${ }^{13}$.

Las claves para el manejo general se resumen de la siguiente manera: 
1. Cuidados estándar a todo paciente con BQ: Kinesiterapia diaria como fundamental, vacuna influenza y neumococo, manejo de co-morbilidades y enfermedad de base, rehabilitación pulmonar, tratamiento precoz de las exacerbaciones, vigilancia del esputo para $P$. aeruginosa y micobacteriosis.

2. En bronquiectasias moderadas o si hay síntomas persistentes pese a los cuidados estándar se recomienda considerar uso de macrólidos en pacientes exacerbadores frecuentes, dispositivos de fisioterapia y nebulizaciones de solución salina hipertónica.

3. En bronquiectasias severas considerar además: antibióticos inhalados cuando existe colonización por $P$. aeruginosa, corticoides inhalados en pacientes seleccionados y oxígenoterapia, cirugía y trasplante pulmonar en indicaciones específicas.

\section{Corticoides inhalados}

En un estudio de BQ no FQ el empleo de altas dosis de corticoides inhalados reduce el volumen del esputo y los marcadores inflamatorios, pero no disminuye las exacerbaciones ni modifica la función pulmonar. Por lo tanto, el uso rutinario de corticoides inhalados no está recomendado, excepto cuando hay hiperreactividad bronquial o una ABPA.

\section{Macrólidos}

El efecto de los macrólidos en BQ se fundamente en su papel anti infeccioso, sus propiedades antiinflamatorias y su efecto inmunomodulador para alterar la virulencia bacteriana. Sabemos que la azitromicina es ampliamente recomendada en fibrosis quística, especialmente en pacientes colonizados con $P$. aeruginosa.

Hay tres estudios multicéntricos que avalan el uso de macrólidos en BQ no FQ: EMBRACE ${ }^{14}$, BAT $^{15}$ y BLESS ${ }^{16}$, los que usando diferentes dosis de azitromicina (500 $\mathrm{mg}$ tres veces a la semana, $250 \mathrm{mg}$ una vez al día) o eritromicina (400 mg dos veces al día) han demostrado reducir las exacerbaciones en estos pacientes.

Los beneficios de los macrólidos deben ser balanceados contra su potencial efecto tóxico cardiovascular y su ototoxicidad y el desarrollo de resistencia de micobacterias (en BQ no FQ debe hacerse exámenes previos para descartar esta infección).

\section{Técnicas de clearance mucociliar}

Las técnicas de drenaje son variadas: técnicas de respiración, drenaje postural, autodrenaje (técnicas activas de respiración y drenaje asistido), técnicas dependientes de dispositivos: PEP o presión espiratoria positiva oscilatoria, chalecos vibratorios, dispositivos de tos asistida; y finalmente ejercicio y rehabilitación pulmonar como estrategias de drenaje ${ }^{17}$.

Técnicas de drenaje a baja espiración:

- Autodrenaje: se instruye al paciente para respirar volúmenes bajos en reserva espiratoria y altos volúmenes en reserva inspiratoria con glotis abierta.

- ELTGOL: (slow-expiration with glotis-openedin-lateral-posture): se le instruye a exhalar lentamente en posición lateral con glotis abierta $^{18}$.

\section{Dispositivos de drenaje vía aérea}

1. Presión Espiratoria Positiva (PEP): oscilatoria o no oscilatoria. Están indicados en pacientes con BQ FQ o no FQ con pobre adherencia a técnicas manuales (Flutter y Accapella) ${ }^{13,19}$.

2. Chaleco vibratorio: chaleco inflable conectado a un generador de pulsos de aire que hace que se infle y desinfle rápidamente, produciendo compresión periódica de la pared torácica en un proceso llamado oscilación de alta frecuencia (HFCWO). Se utiliza para BQ FQ, BQ no FQ, desórdenes ventilatorios, desórdenes neuromusculares, atelectasias y EPOC. Requiere de tos eficiente $^{20}$.

\section{Terapia mucolítica e hiperosmolar}

Existen varias terapias mucolíticas aprobadas para su uso en bronquiectasias no FQ:

1. Nebulización con solución salina hipertónica 7\%: muestran resultados mixtos, en algunos estudios con mejoría de calidad de vida, aumento de la motilidad ciliar, aumento del clearance de la tos. Sin embargo, aunque otros no encontraron grandes diferencias en comparación con placebo, aun así se recomienda su uso en estos pacientes ${ }^{21}$.

2. Manitol: Estudios recientes muestran mejoría en el tiempo a la siguiente exacerbación y mejoría calidad de vida medido por SGRQ (St. George Respiratory Questionnaire $)^{22}$. 
3. DNasa recombinante: que es una terapia indispensable en pacientes con FQ, no está recomendada en BQ no FQ. En las Guías Británicas de 2010 se describe una recomendación tipo A en contra del uso de DNasa para bronquiectasia no $\mathrm{FQ}^{7}$. O'Donnell y cols., estudiaron 349 pacientes, los que randomizaron a 173 con DNasa y 176 con placebo. Encontraron una reducción del $\mathrm{VEF}_{1}$ de $-3,6 \%$ versus $1,7 \%, p<0,05$ en contra del primer grupo, con un riesgo relativo de aumento de las exacerbaciones de 1,35 (IC: 1,01-1,79) . $^{23}$.

\section{Disminución de la infección bacteriana}

El objetivo primario del tratamiento de las BQ es disminuir las infecciones y la colonización bacteriana, y con ello reducir las exacerbaciones, mejorar la calidad de vida, reducir los síntomas, mejorar la función pulmonar, prevenir hospitalizaciones y disminuir la mortalidad. Para lograr reducir la infección bacteriana hay que enfrentar dos situaciones: tratar las exacerbaciones y manejar la infección crónica.

\section{Tratamiento de la exacerbación}

Se define como exacerbación el aumento de la tos, aumento del esputo, el cambio de viscosidad o aumento de purulencia, el aumento de la disnea y/o la presencia de hemoptisis.

El paciente debiera hospitalizarse por los siguientes motivos: cianosis, confusión, frecuencia respiratoria $>25 \mathrm{ciclos} / \mathrm{min}$, falla respiratoria o hemodinámica, temperatura $>38^{\circ} \mathrm{C}$, incapacidad de tratamiento oral, fracaso a terapia oral y razones sociales.

El uso de cefalosporina con efecto antipseudomónico más un aminoglicósido o bien una quinolona, especialmente ciprofloxacino, es una muy buena combinación para erradicar el germen. También se ha usado ciprofloxacino oral en altas dosis más aminoglicósido inhalado como alternativa en pacientes ambulatorios.

En una exacerbación el tratamiento inicial es empírico y debe ser guiado por el último cultivo realizado. Previo al inicio de los antibióticos se debe solicitar un cultivo de expectoración, pero iniciar tratamiento empírico en espera de resultados, ya que el antibiótico se puede modificar una vez obtenido el antibiograma.

Los mejores resultados se obtienen con altas dosis de antibióticos. Los microorganismos asociados con las exacerbaciones son $H$. influenzae, S. pneumoniae, M. catarrhalis, S. aureus sensible, $S$. aureus resistente, Coliformes y $P$. aeruginosa ${ }^{7}$.

El germen más frecuente es el $H$. influenzae y el tratamiento con beta-lactámicos es suficiente: amoxicilina en dosis altas ( $1 \mathrm{~g}$ cada $8 \mathrm{~h})$ o bien amoxicilina-ácido clavulánico cada $12 \mathrm{~h}$. La $P$. aeruginosa, si es sensible y está colonizando previamente, debe tratarse con una quinolona oral, preferentemente ciprofloxacino, $750 \mathrm{mg}$ dos veces al día. No hay consenso en la duración de la terapia, pero la recomendación es de 10 a 14 días de tratamiento.

Debemos iniciar un tratamiento intravenoso si el paciente se encuentra comprometido, requiere hospitalización, no responde a la terapia oral, el microorganismo es resistente o se pretende erradicar un primer cultivo con $P$. aeruginosa.

Un tratamiento agresivo de una colonización inicial por $P$. aeruginosa puede resultar en una erradicación del organismo y así prevenir o retardar una nueva colonización. La erradicación de $P$. aeruginosa ha demostrado además reducir las exacerbaciones.

Existen pocos estudios sobre erradicación precoz de $P$. aeruginosa en BQ no FQ. Orriols y cols., estudiaron 34 pacientes con cultivos positivos, tratados con ceftazidima más tobramicina IV durante 14 días seguido de 3 meses de tobramicina nebulizada o de placebo, y controlados por 12 meses. Con la tobramicina nebulizada se obtuvo una mayor proporción de pacientes libres de $P$. aeruginosa a los 12 meses (54,5\% versus 29,4\%) en comparación con el grupo placebo ${ }^{24}$.

\section{Terapia combinada o monoterapia para P. aeruginosa}

Existe controversia respecto a utilizar monoterapia o terapia combinada. El Consenso Europeo del año 2000 concluye que, con un cultivo sensible, la monoterapia puede ser tan efectiva como la terapia dual. Por otra parte, en presencia de un cultivo con $P$. aeruginosa resistente, la terapia combinada es la terapia de elección, bajando el riesgo de desarrollar más tarde resistencia a los otros antibióticos ${ }^{25}$. La recomendación para la combinación es una cefalosporina de tercera generación con efecto antipseudomónico como ceftazidima más un aminoglicósido como amikacina dosis diaria por 14 días.

La terapia combinada no es necesaria en otros gérmenes, salvo excepciones.

Las guías británicas de 2010 recomiendan que algunos pacientes con $\mathrm{BQ}$ pueden responder a un antibiótico aun siendo resistente a esta droga in 
vivo, y en este caso no se debe cambiar el antibiótico si la respuesta clínica es favorable?

En relación a las terapias antibióticas inhaladas de larga duración Brodt y cols., publicaron una revisión sistemática incluyendo 12 estudios randomizados controlados con 1.264 pacientes, encontrando que los antibióticos (amikacina, aztreonam, ciprofloxacino, colistin, gentamicina y tobramicina) usados durante 1 a 12 meses eran más efectivas que placebo en disminuir carga bacteriana, erradicar bacterias y disminuir exacerbaciones ${ }^{26}$. Murray y cols., en un estudio publicado en 2011 demuestran que las nebulizaciones con gentamicina en BQ noFQ en el $31 \%$ se lograba erradicación de $P$. aeruginosa, había un aumento significativo en el tiempo a la siguiente exacerbación y una disminución de las exacerbaciones ${ }^{27}$.

\section{Trasplante pulmonar}

Hoy día el trasplante bipulmonar es una opción para pacientes con BQ no FQ en la etapa final de su enfermedad, tanto en niños como en adultos. En las guías internacionales para la selección de candidatos a trasplante pulmonar de ATS/ERS se debe referir a un equipo de trasplante a los pacientes menores de 60 años, con $\mathrm{VEF}_{1}<30 \%$, o si tienen un deterioro muy rápido en el último año pese a un óptimo manejo médico, en pacientes con hemoptisis masiva, hipertensión pulmonar secundaria severa, o admisión a UCI (unidad de cuidados intensivos) ${ }^{28}$.

\section{Bibliografía}

1.- http://ec.europa.eu/health/rare_diseases/policy/registries/index_en.htm (consultado el 30 de mayo de 2016).

2.- MCSHANE P J, EDWARD T. NAURECKAS E T, GREGORY TINO G, MARY E. STREK M E. Non-Cystic Fibrosis Bronchiectasis. Amer J Respir Crit Care Med 2013; 188: 647-56.

3.- GOEMINNE P C, DE SOYZA A. Bronchiectasis: how to be an orphan with many parents? Eur Respir J 2016; 47: $10-3$.

4.- CHALMERS J D, GOEMINNE P C, ALIBERTI S. The bronchiectasis severity index: an international derivation and validation study. Am J Respir Crit Care Med 2014; 189; 576-85.

5.- MARTÍNEZ-GARCÍA M, DE GRACIA J, VENDRELL M, GIRÓN R M, MÁIZ L, DE LA ROSA D, et al. Multidimensional approach to non-cystic fibrosis bronchietasis: the paced score. Eur Respir J 2014; 43: 1357-67.
6.- GUAN W, CHEN R, ZHONG N. The bronchiectasis severity index and faced index for bronchiectasis. Eur Respir J 2016; 47; 382-4.

7.- PASTEUR M C, BILTON D, HILL A T, on behalf of the British Thoracic Society Bronchiectasis (non-CF) Guideline Group. British Thoracic Society guideline for non-CF bronchiectasis. Thorax 2010; 65: i1-i58.

8.- FINCH S, MCDONNELL M J, ABO-LEYAH H, ALIBERTI S. A Comprehensive Analysis of the Impact of Pseudomonas aeruginosa Colonization on Prognosis in Adult Bronchiectasis. Ann Am Thorac Soc 2015; 12 : 1602-11.

9.- BONAITI G, PESCI A, MARRUCHELLA A, LAPADULA G, GORI A, ALIBERTI S. Nontuberculous Mycobacteria in Noncystic Fibrosis Bronchiectasis. Biomed Res Int 2015; 2015: 197950.

10.- ALIBERTI S, MASEFIELD S, POLVERINO E, DE SOYZA A, LOEBINGER M R, MENÉNDEZ R, et al. Research priorities in bronchiectasis: a consensus statement from the EMBARC Clinical Research Collaboration. ERJ Express. Published on June 10, 2016 as doi: 10.1183/13993003.01888-2015.

11.- KEISTINEN T, SÄNÄJÄKANGAS O, TUUPONEN T, KIVELÄ S L. Bronchiectasis: an orphan disease with a poorly-understood prognosis. Eur Respir J 1997; 10: 2784-87.

12.- LEE A, HILL C J, MCDONALD C F, HOLLAND A E. Arch Phys Med Rehabil 2016 Jun 16. pii: S0003-9993 (16). doi: 10.1016.

13.- LEE A, BURGE A T, HOLLAND A E. Pulmonary rehabilitation in individuals with non-cystic fibrosis bronchiectasis: A systematic review. Cochrane Database Syst Rev 2015. Nov 23 (11) CD086341. doi10.1002/14651858 CD008351. pub

14.- WONG C, JAYARAM L, KARALUS N, EATON T, TONG C, HOCKEY H, et al. Azithromycin for prevention of exacerbations in non-cystic fibrosis bronchiectasis (EMBRACE): a randomised, double-blind, placebo-controlled trial. Lancet 2012; 380: 660-7.

15.- FAN L C, LU H W, WEI P, JI X B, LIANG S, XU J F. Effects of long-term use of macrolides in patients with non-cystic fibrosis bronchiectasis: a meta-analysis of randomized controlled trials. BMC Infectious Diseases 2015; $15: 160$.

16.- ROGERS G B, BRUCE K D, MARTIN M L, BURR L D, SERISIER D J. The effect of long-term macrolide treatment on respiratory microbiota composition in non-cystic fibrosis bronchiectasis: an analysis from the randomised, double-blind, placebo-controlled BLESS trial. Lancet Respir Med 2014; 2: 988-96.

17.- MCILWAINE M, BUTTON B, DWAN K. Positive expiratory pressure physiotherapy for airway clearance in people with cystic fibrosis. Cochrane Database of Systematic Reviews 2015, Issue 6. Art. No.: CD003147.

18.- MAIN E, GRILLO L, RAND S. Airway clearance strategies in cystic fibrosis and non-cystic fibrosis 
bronchiectasis. Semin Respir Crit Care Med 2015; 36 : 251-6.

19.- REYCHLER G, JACQUEMART M, PONCIN W, AUBRIOT A S, LIISTRO G. Benefit of educational feedback for the use of positive expiratory pressure device. Braz J Phys Ther 2015; 19: 451-6.

20.- NICOLINI A, CARDINI F, LANDUCCI N, LANATA S, FERRARI-BRAVO M, BARLASCINI C. Effectiveness of treatment with high-frequency chest wall oscillation in patients with bronchiectasis. BMC Pulmonary Medicine 2013; 13: 21-8.

21.- KELLETT F, ROBERT N M. Nebulised 7\% hypertonic saline improves lung function and quality of life in bronchiectasis. Respir Med 2011; 105: 1831-5.

22.- BILTON D, TINO G, BARKER A F, CHAMBERS D C, DE SOYZA A, DUPONT L J A, et al. Inhaled mannitol for non-cystic fibrosis bronchiectasis: a randomised, controlled trial. Thorax 2014; 69: 1073-9.

23.- O'DONNELL A E, BARKER A F, ILOWITE J S, FICK R B. Treatment of Idiopathic Bronchiectasis With Aerosolized Recombinant Human DNase I. rhDNase Study
Group. Chest 1998; 113: 1329-34.

24.- ORRIOLS R, HERNANDO R, FERRER A, TERRADAS S, MONTORO B. Erradication Therapy against Pseudomonas aeruginosa in Non-Cystic Fibrosis Bronchiectasis. Respiration 2015; 90: 299-305.

25.- DÖRING G, CONWAY S P, HEIJERMAN H G, HODSON M E, HØIBY N, SMYTH A, et al. Antibiotic therapy against Pseudomonas aeruginosa in cystic fibrosis: a European consensus. Eur Respir J 2000; 16: 749-67.

26.- BRODT AM, STOVOLD E, ZHANG L. Inhaled antibiotics for stable non-cystic fibrosis bronchiectasis: a systematic review. Eur Respir J 2014; 44: 382-93.

27.- MURRAY M P, GOVAN J R, DOHERTY C J, SIMPSON A J, WILKINSON T S, CHALMERS J D, et al. A randomized controlled trial of nebulized gentamicin in non-cystic fibrosis bronchiectasis. Am J Respir Crit Care Med 2011; 183: 4: 491-49.

28.- INTERNATIONAL GUIDELINES FOR THE SELECTION OF LUNG TRANSPLANT CANDIDATES. Am J Respir Crit Care Med 1998; 158: 335-9.

Correspondencia a:

Dra. Patricia Fernández V.

General Salvo 357, Providencia, Santiago.

Email: pafeva99@hotmail.com 